\title{
Spontaneous pneumomediastinum: is it a rare cause of chest pain?
}

\author{
ALON YELLIN, MARKUS GAPANY-GAPANAVICIUS, YAIR LIEBERMAN \\ From the Department of Thoracic Surgery, Chaim Sheba Medical Centre, and Tel Aviv University, Sackler \\ School of Medicine, Tel Hashomer, Israel
}

ABSTRACT A policy of obtaining a routine chest radiograph in every young patient with unexplained chest pain or dyspnoea led to the diagnosis of 14 cases of spontaneous pneumomediastinum within four years, whereas only two cases from former years could be found retrospectively. One patient had recurrent spontaneous pneumomediastinum, the first case so far reported. Analysis of admission data suggested that this entity may be much more common than is generally believed, and may be second only to spontaneous pneumothorax as a cause of admission of young, healthy individuals experiencing sudden chest pain or shortness of breath. After reviewing the published reports we propose the following definition of spontaneous mediastinal emphysema: the non-traumatic presence of free air in the mediastinum in a patient with no known underlying lung disease. When it occurs without associated pneumothorax it is benign. The incidence of spontaneous pneumomediastinum for the four-year period under study was 1 per 12850 admissions.

The occurrence of free air in the mediastinum is known as mediastinal emphysema or pneumomediastinum. There are several routes by which air may gain access to the mediastinum such as perforations of the oesophagus, trachea, and bronchi, or via the retroperitoneum after perforation of an abdominal viscus. Air can also enter through wounds caused by tracheostomy, ${ }^{1}$ cervical and thoracic injuries, operative procedures in the neck and buccal cavity, or high-speed air turbine dental drills during tooth extraction. ${ }^{2}$ The most frequent cause is non-traumatic rupture of marginal pulmonary alveoli, allowing air to travel along interstitial and vascular routes. ${ }^{3}$ This variety, known as medical mediastinal emphysema, ${ }^{4}$ includes a subgroup called spontaneous pneumomediastinum, in which no underlying disease is demonstrable (first described by Hamman in $1939^{5}$ ). It is generally considered to be uncommon, ${ }^{46}$ although Millard, finding four cases in his group within nine months, suggested that it may be more prevalent than has been reported. ${ }^{7}$

\footnotetext{
Address for reprint requests: Dr Alon Yellin, Department of Thoracic Surgery, Sheba Medical Centre, Tel Hashomer 52621, Israel.
}

Accepted 21 January 1983
In January 1978 we admitted a patient with chest pain who had no abnormal clinical signs but a pneumomediastinum on the chest radiograph. We therefore proposed that chest radiography should be performed routinely on every young person seen in the emergency room for unexplained chest pain or shortness of breath. We have diagnosed 13 cases of spontaneous pneumomediastinum from 1978 to 1981 , since this policy was put into effect.

\section{The patients}

All cases of spontaneous pneumomediastinum have been followed up since January 1978 . We searched hospital records for cases before 1978 and examined the case notes. There were 16 patients, 14 male and two female (table), of whom 14 were seen after January 1978. Only two earlier cases were found, one occurring in 1966 and the other in 1974. The annual occurrence was as follows: 1978 -three cases (1 per 13744 admissions); 1979 -two (1 per 20 032); 1980 -three (1 per 15 911); 1981-seven cases (1 per 7286 ). The chief complaint on admission was retrosternal pain in 10 patients, cervical pain in one patient, and shortness of breath in five. Five patients had mild-to-moderate dysphagia.

Factors that could have contributed to the immediate pathogenesis of pneumomediastinum 
Summary of clinical data on the 16 patients (14 male, two female)

\begin{tabular}{ll}
\hline Age (years) & $14-29$ \\
Mean & 20 \\
Chief complaint & 10 \\
Chest pain & 1 \\
Cervical pain & 5 \\
Dyspnoea & 6 \\
Possible precipitating factor & 2 \\
Upper respiratory tract infection & 2 \\
Non-specific cough & 2 \\
Chlorine exposure & 4 \\
Asthmatic attack & \\
Physical exertion & 4 \\
Other disease & 1 \\
Asthma & 8 \\
Epilepsy & 9 \\
Physical findings & 5 \\
Cervical crepitations & 16 \\
Mediastinal crunch & $2-5$ \\
None & $3 \cdot 3$ \\
Mediastinal air on chest radiograph & $6-52$ \\
Period in hospital (days) & 22 \\
Mean & 1 \\
Follow-up (months) & \\
Mean & \\
No with recurrence &
\end{tabular}

were found in all patients. These included upper respiratory infection in six patients, non-specific cough in two, severe cough after exposure to chlorine gas in two, physical exertion in four, an asthmatic attack in one, and dental drilling in one. Four patients had bronchial asthma, though only one presented during an acute attack and two had not experienced asthmatic symptoms for at least six years. One patient had epilepsy, which was well controlled.

On examination all patients, apart from those suffering from an additional condition, were healthy, but they showed various degrees of anxiety. Subcutaneous emphysema could be palpated in eight patients and Hamman's sign (mediastinal "crunch") was present in nine. In five patients physical examination showed nothing abnormal. Diagnosis was established in all cases by a chest radiograph that showed paracardiac and mediastinal free air and, in most cases, air in the anterior cervical and bilateral supraclavicular regions. Three patients with a synchronous pneumothorax and two admitted initially for a pneumothorax and found to have mediastinal emphysema on a later radiograph were excluded from this series. The first patient in the series and the first patient exposed to chlorine had a barium meal. Blood gas analysis was performed sporadically and usually gave normal results.

All patients were observed in the hospital for two to five days (mean 3.3 days). No specific treatment was given. Complete resorption of mediastinal air occurred in four to eight days.

The 14 patients seen after January 1978 were followed up for six to 52 months. One patient experi- enced a recurrence of pneumomediastinum 14 months after the first episode.

\section{Discussion}

Spontaneous pneumomediastinum was first reported in 1939 by Hamman..$^{5}$ Bodey ${ }^{4}$ reported an incidence of one per 42000 admissions. Munsell reported one case per 7115 admissions in a United States Air Force hospital, mainly serving the age group in which spontaneous mediastinal emphysema is liable to occur. The incidence of 1800 admissions reported by McMahon $^{8}$ is considerably higher, although the cases occurred in a naval hospital. The present series consists of 16 cases, 14 of which were diagnosed from January 1978 to December 1981 in one general hospital. The increased incidence after 1978 (1:12 850) might be due to increased awareness of the clinicians and radiologists. For patients aged 14-29 the incidence was 1:3000, and for the 5150 non-surgical, non-traumatic, and nonobstetric patients admitted in this age group it was $1: 368$. The number of patients aged 14-29 admitted for sudden chest pain or shortness of breath was much lower, and for them spontaneous pneumomediastinum was second only to spontaneous pneumothorax as a cause of admission.

During 1978-81 there were 210 admissions for spontaneous pneumothorax, so the ratio of spontaneous mediastinal emphysema to spontaneous pneumothorax was $1: 15$. The similar sex distribution-1 F:7 M for spontaneous pneumomediastinum compared with 1:8 for spontaneous pneumothorax - would be consistent with a common aetiology. The suggestion that spontaneous pneumomediastinum is due to increased alveolar pressure, proposed by Macklin and Macklin ${ }^{3}$ and challenged by Morere et al,${ }^{9}$ is accepted by most authors. The sudden increase in intrabronchiolar pressure is usually initiated by coughing or sudden expiration against a closed glottis. Spontaneous pneumothorax may have a similar pathogenesis, although most patients have subpleural blebs and therefore have a high recurrence rate $(35 \%$ in our series). Patients with spontaneous pneumomediastinum probably have normal lung parenchyma. Our patients had a mean follow-up of 22 months, during which time there was one recurrence of pneumomediastinum. So far as we know, it is the first case of recurrent mediastinal emphysema to be published.

The exact definition of spontaneous pneumomediastinum is controversial. Some reports include patients with underlying pulmonary diseases, such as hyaline membrane disease ${ }^{6}$ or pneumonia. $^{10}$ We agree with McMahon $^{8}$ that the 
name spontaneous pneumomediastinum should not be used for such patients, but we would include young asthmatic patients with preserved pulmonary function. One of the patients in McMahon's series ${ }^{8}$ and five in Munsell's report ${ }^{6}$ had a pneumothorax; such cases were excluded from our series. We propose that the term spontaneous pneumomediastinum should be reserved for patients with no underlying pulmonary disease who have a non-traumatic, mediastinal air leak. We have included two cases of pneumomediastinum occurring after inhalation of chlorine, ${ }^{11}$ as we believe that the severe coughing and not the chlorine per se was the pathogenic factor. During the same period we observed five male patients, aged 18-21, with isolated "traumatic" pneumomediastinum. Two had been in a minor road traffic accident, two had been in fights, and one had been exposed to high oxygen pressure in a testing device. All presented with chest pain or shortness of breath and physical and radiographic examination excluded other injuries. Although we believe that pneumomediastinum after these traumas evolved in the same manner as in the spontaneous variety we have excluded traumatic cases from this series.

The outcome in patients with spontaneous mediastinal emphysema was very favourable and no treatment was necessary. Patients spent a mean of 3.3 days in hospital and had complete resorption of mediastinal air within eight days. Dysphagia, which may be the chief complaint, ${ }^{12}$ was experienced by five patients including the two exposed to chlorine. ${ }^{11}$ Although this symptom can be alarming, oesophageal examination is not essential. We suggest that a young, healthy person admitted with sudden chest pain or shortness of breath, with free air in the mediastinum but no pneumothorax, requires only observation.
Our special thanks are due to Dr Gerald L Baum for his kind advice when we were preparing this manuscript.

\section{References}

${ }^{1}$ Han SY, Rudolph AJ, Teng CT. Pneumomediastinum in infancy. J Pediatr 1963;62:754-61.

${ }^{2}$ Hunt RB, Sahler OD. Mediastinal emphysema produced by air turbine dental drills. JAMA 1968;205:241-2.

${ }^{3}$ Macklin MT, Macklin CC. Malignant interstitial emphysema of the lungs and mediastinum as an important occult complication in many respiratory diseases and other conditions: interpretation of clinical literature in light of laboratory experiment. Medicine (Baltimore) 1944;23:281-358.

${ }^{4}$ Bodey GP. Medical mediastinal emphysema. Ann Intern Med 1961;54:46-56.

${ }^{5}$ Hamman L. Spontaneous mediastinal emphysema. Bull Johns Hopkins Hosp 1939;64:1-21.

${ }^{6}$ Munsell WP. Pneumomediastinum. JAMA 1967;202:689-93.

${ }^{7}$ Millard CE. Pneumomediastinum. Chest 1969;56:297300.

${ }^{8} \mathrm{McMahon}$ DJ. Spontaneous pneumomediastinum. Am J Surg 1976;131:550-1.

${ }^{9}$ Morere P, Fleury J, Vandour P, Bohu D, Evreux P, Stein JP. Spontaneous microtraumatic mediastinal emphysema in children with bronchial asthma. Presse Méd 1966;74:1953-6.

${ }^{10}$ Gray JM, Hanson GC. Mediastinal emphysema: aetiology, diagnosis and treatment. Thorax 1966;21:32531.

${ }^{11}$ Gapany M, Yellin A, Almog S, Tirosh M. Pneumomediastinum - a complication of chlorine exposure from mixing household cleaning agents. JAMA 1982;248:349-50.

${ }^{12}$ Shuster MJ. Pneumomediastinum as a cause of dysphagia and pseudodysphagia. Ann Emerg Med 1981;10:648-51. 